\title{
Getting back to exercise without pain: The core
}

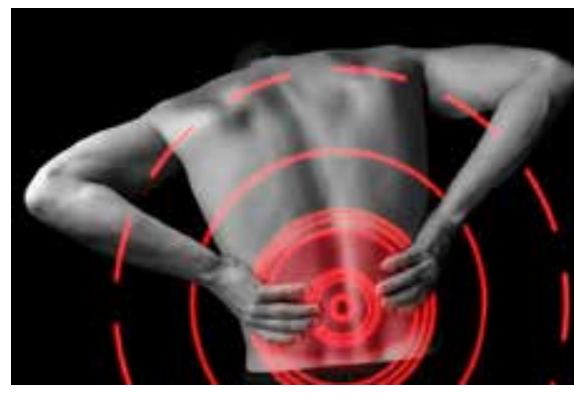

Abstract

Core strengthening also referred to as lumbar stabilization is used as a therapeutic exercise treatment for low back pain (LBP). In those with LBP, growing evidence suggest, that the structure and function of the deep stabilizers within the core such as the transversus abdominis ( $\operatorname{Tr} A$ ) and lumbar multifidus (LM) are altered (Hodges-Richardson, 1996; O'Sullivan, et al., 1997; Tsao-Hodges, 2008). These muscles normally contract in an anticipatory manner to limb movement in healthy people Hodges-Richardson, 1996, 1998; Ferreira et al., 2004; Hebert et al., 2010) and provide a stiffen spine segment needed for accurate limb movement (O'Sullivan et al., 1997).

By targeting the TrA and the LM simultaneously (Richardson-Jull, 1995; Hebert et al., 2010) in LBP patients and clients researchers saw improved strength, endurance, motor control in these muscles, improved sport performance in general and pain was successfully alleviated (Richardson-Jull, 1995; Tsao-Hodges, 2008; Akuthota et al., 2008).

As a personal trainer, I have been taught that the core should be activated before the vigorous movement of training, that it is undertaken by either 'hollowing in' or bracing the belly button to activate the stabilizers of the spine. Research suggest that by regularly engaging the core muscles with first hollowing then later bracing clients and patients can learn to turn on and improve motor control of the TrA muscle.

Key words: lumbar stabilization, low back pain, deep trunk stabilizers, transversus abdominis, core strengthening

\section{Introduction}

It has become evident from the previous articles in this series on musculoskeletal disorders that problems are rarely located at the site of the pain unless the injury is caused by a collision or sudden blow. Upon examination of the chain of muscles in which the pain is situated, one generally discovers the root cause of the issue lies with muscles further down or up the line or directly opposite (crossed syndromes) from the aggrieved area. It is recommended that professionals look to a more systematic, holistic and 'whole body' approach when looking for the cause of the musculoskeletal imbalance and pain.

As discussed in previous articles, as trainers, we look to static and dynamic postures to identify postural deviations that originate from muscular imbalances. For a trained eye, it is easy to see - from a standing posterior view - how someone complaining of a right tennis elbow may also be affected by a dropped right shoulder and a pelvis that sits high on the same side. This posture, caused by the altered biomechanics in the upper body, then results in a tennis elbow complaint - surprising those clients who don't even play a racquets sport!

As previously explained, with lower back, knee and ankle pain, there were typical muscles that have become weak and underactive alongside those that have become overactive and tight. As many other personal trainers will testify, this 'tightness/ weakness' combination - which is seen repeatedly among clients - has developed in response to certain habitual repeated behaviors. A typical musculoskeletal imbalance of the lower body is the Lower Crossed Syndrome (LCS) that is characterized by tight hip-flexors that are pulling the pelvis into an excessive anterior tilt, which in turn can cause the abdominals and gluteals to lengthen and weaken permanently.

Despite undertaking a well-planned deactivation/activation exercise continuum some clients with LCS or LBP may deviate back to previous movement patterns, weaknesses and the lack of progress in their movements in the gym suggests that progress is hindered.

The so-called core is the group of trunk muscles that surround the spine and abdominal viscera. The core local muscles include the transverse abdominis, multifidus, internal obliques, transversospinalis, and pelvic floor muscles; the core global muscles include the erector spinae, external obliques, rectus abdominis, and quadratus lumborum (Akuthota-Ferreiro, 2008). Core strengthening, often referred to as lumbar stabilization training. Core stability or lumbar stability is defined as the ability to control the position and motion of the trunk over the pelvis to allow optimum production, transfer and control of force to the limbs in integrated functional and athletic activities. Core muscle activity is best understood as the pre-programmed integration of local, single-joint muscles and global multi-joint muscles to provide stability for motion (Kibler, 2006,p190).

Core stability programs main aim is to utilize both the lumbar multifidus and the transversus abdominis at the same time (Hebert et al., 2010). Research supports that targeting $\operatorname{TrA}$ and $\mathrm{LM}$ is a superior method to any other strengthening program when presented with niggles and pains or the LCS. Strengthening the superficial abdominal muscles such as the external oblique and rectus abdominis does not improve TrA activation capacity and activation hence core strength (Franca et al. 2010), but working on TrA endurance has a positive effect on the work capacity of the superficial muscles therefore the training of the TrA should precede the training of the other abdominal muscles. Core stability training can provide remedy to the problem of inadequate movement via improving deep lumbar/ segmental stabilization, muscle endurance and motor control via the central nervous system (CNS) (Pereira et al. 2011, Panjabi, 1992) ultimately providing a stiff base for movement to occur. Without the engagement of the local core muscles in the required timely manner - even a client who exercises regularly has a visibly toned and athletic body - can encounter problems in the lower back and distal joints in a seemingly unrelated manner.

\section{Questions}

1) Is the TrA one - if not the most important - abdominal muscles to observe when coaching clients, particularly so when lifting from the floor or performing stabilizing moves with the trunk?

2) Can we teach this muscle to turn on on time before limb movement or before unknown functional tasks?

\section{Deep Stabilizers}

$\operatorname{Tr} A$ is one of the main core stabilizing muscles of the lumbar spine (Cresswell et al., 1992). Studies show that a weak TrA muscle may be to blame for low back pain (Hodges-Richardson 1996,1998b,1999; O'sullivan et al., 1997; Ferreira et al., 2004; Tsao-Hodges, 2008).

The TrA is the deepest layer of abdominal muscle and runs between the ribs and the pelvis, and the lateral raphe of the thoracolumbar fascia, a weave of connective tissue, and the linea alba horizontally from front to back (Figure 2). 


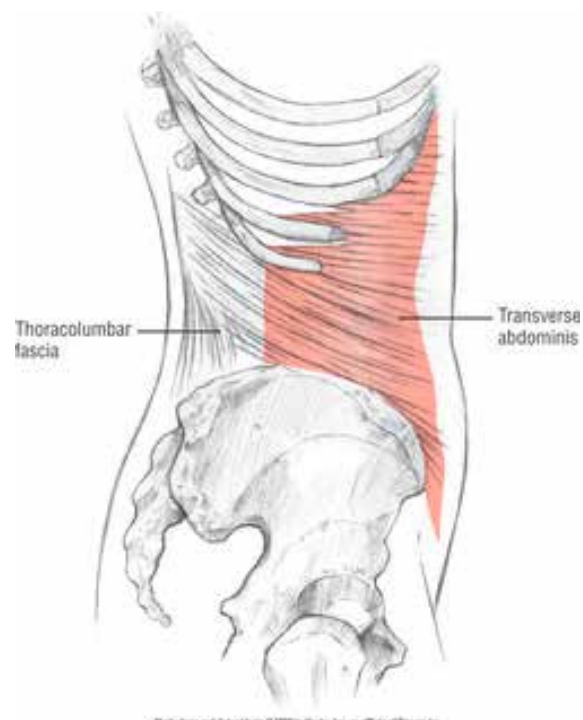

Figure 2: Transversus abdominis anatomy

It appears from EMG studies that the TrA is constantly activated prior to other trunk muscles (such as the internal oblique (OI), external oblique (OE), rectus abdominis (RA) and the erector spinae (ES) (Hodges \& Richardson 1997b). When activated, the TrA muscles create a deep 'corset', its activation flattens the abdominal wall, compresses the viscera (internal organs), generating pressure without causing trunk motion; concurrently the diaphragm and the pelvic floor muscles get activated which together create an abdominal 'canister' which is necessary for the TrA to increase intra-abdominal pressure (IAP) and tension in the thoracolumbar fascia (TLF) (Cresswell et al, 1992,1994), hence contributes to the stiffening process that occurs before limb movement (Hodges-Richardson 1997a,1998) (Figure 3).

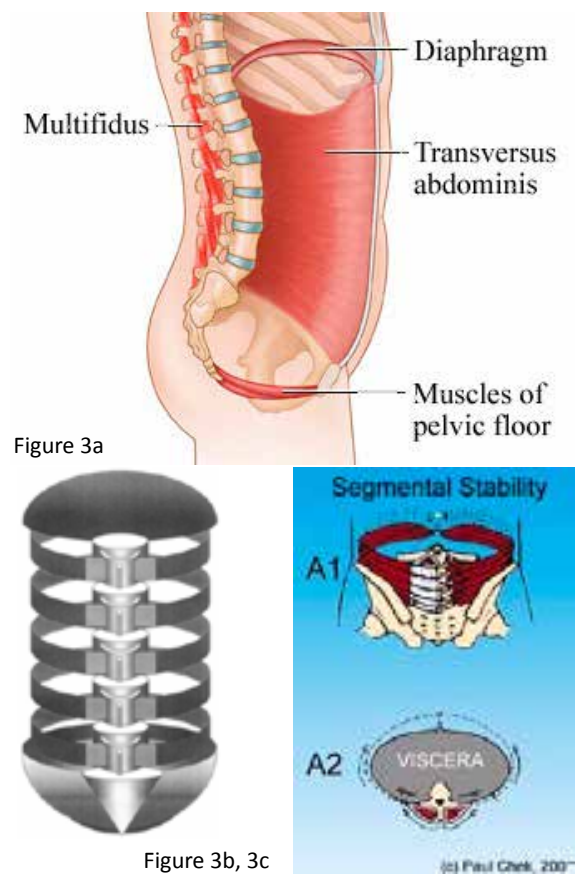

Muscular (3a) and diagrammic (3b) representation of the abdominal 'canister' formed by co-activation of the diaphragm, transversus abdominis and the pelvic floor. The deep 'corset' that is created by the contraction of the TrA.

From these studies, we now understand that the major function of the TrA muscle is to stabilize the spine during movements that involve the arms and legs - and to increase via the TLF the extension forces that keep the spine upright. Movement examples where the TrA's engagement can be observed include descending in a weighted squat exercise / deadlift or lifting an object from the floor up (Cresswell- Thortensson, 1994) (Figure 4).

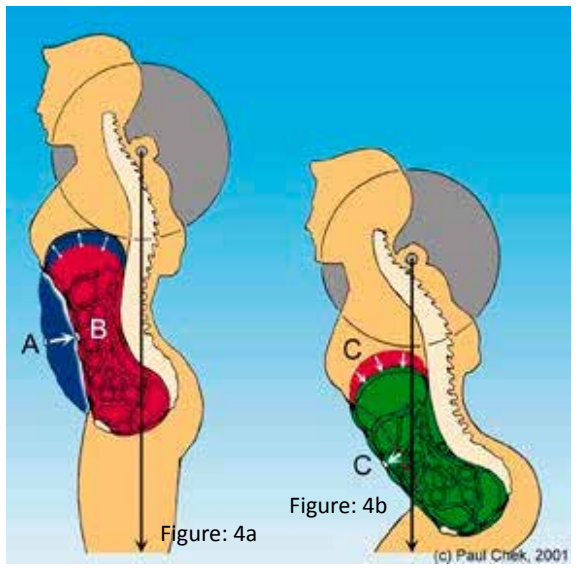

Figure 4: Spine stabilization and deep abdominal wall activation

As the TrA contracts against the viscera (4a) the organs pushed superiorly against the diaphragm and inferiorly into the pelvic floor. As the squatter descends and draws air in that charges the thoracic cavity pushes the diaphragm down (4b). The intra-abdominal pressure increases even further. The TrA is under great eccentric load, might be pushed forward under heavy load but under submaximal load the abdomen should be staying flat.

\section{Transversus abdomin- is relationship to load}

The behavior of the TrA has been well-documented with studies using fine wire and surface EMG (Cresswell-Thortensson, 1994, Hodges-Richardson, 1997). The response in the IAP occurs in a two-burst pattern, which is consistent with the response of the TrA during rapid movement with greater magnitude at the start of movement, and then with a less intensity of activation throughout movement (Hodges, 1999). It has been argued that the TrA has a tonic activity in standing when at rest this is possibly related to the gravitational forces of the abdominal contents (DeTroyer et al., 1990), while lying supine the muscle is relaxed (DeTroyer, 1983).

Also it was found that magnitude of activation is greatest with the fastest movement speeds (Cresswell-Thorstensson, 1994; Hodges-Richardson, 1999). However from the studies of Creswell and colleagues 1992, 1993, as well as Hodges and colleagues 1999, 1997b it became evident that the activation of the $\operatorname{TrA}$ is not related neither to the direction of the trunk move- ment, the acceleration or deceleration of the trunk nor the changing forces acting on the spine.

It is anticipated that the TrA contributes to spinal stability by increasing inter-segmental control of the spine. The control is of paramount importance as the lumbar spine is prone to instability and there is a great need that the spine stays in a neutral zone (Panjabi, 1992) (please refer back to article about the lower back). It is now easy to understand how weakness of the TrA causing lack of control of the lumbar segments by failing to increase stiffness of the intervertebral joints that is often manifested in lower back pain. If you're looking to alleviate lower back pain, strengthening your TrA muscle may be the ticket.

\section{Disfunctioning trans- versus abdominis and low back pain (LBP)}

Changes in the automatic control of TrA have been found in people with low back pain (Hodges-Richardson, 1996,1998; Ferreira et al., 2004; Tsao-Hodges, 2008). Also, a delayed onset of its contraction and a loss of its tonic and pre-adjusting function has been hypothesized to result in inefficient muscular stabilization of the spine through a motor control deficit by the CNS (Tsoa-Hodges, 2008; Hodges-Richardson, 1996, 1998; Hodges, 1999).

On top of this, activation of the other abdominal stabilizer muscles also appears delayed, but to a lesser extent (Hodges, 1999). The TrA seems to be vital to gain back appropriate spinal control with the co-contraction of the lumbar multifidus (Richardson-Jull, 1995). As both LM and TrA are local muscles directly attaching to the lumbar spinal segments, they seem to be equally important components of the stabilization system of the lumbar spine, training their co-contraction will be just as important as activating the $\operatorname{Tr} A$ in isolation.

\section{How to retrain the deep abdominal muscle}

Here are few important points:

- The TrA is controlled independently of other trunk muscles and should be trained separately; an isometric exercise is most beneficial for reeducating it to its stabilizing role (Hall et al., 2009).

- TrA has a similar function and level of activation in varying situations and directions; therefore exercise may not need to be initially performed in functional positions to get activation (Hall et al., 2009).

- At a later stage, isometric exercises can 
be combined with dynamic functional exercises involving other body parts of the body. (Richardson-Jull, 1995).

- Co-contraction of the deep abdominal TrA and deep back muscle LM as agonist and antagonist is necessary (O'Sullivan et al., 1997).

- TrA should be trained to contract tonically at a low level $25-40 \%$ maximal voluntary contraction (McArdle et al., 1991).

- The functional interaction between TrA, LM, diaphragm and pelvic floor muscles should be considered.

- As co-contraction of LM and TrA requires specific motor skills for maximal benefit, exercises need to be repeated as many times as possible daily in an initial 8 week period (Richardson-Jull, 1995).

There are generally two ways to activate the TVA muscle for improved core stabilization. The first is with bracing and the second is with hollowing (Figure 5). Yet, optimal and healthy prescription and undertaking of these procedures can often become confused among fitness professionals with their being overprescribed or over-performed such as with excessive hollowing when the client doesn't manage to breath normally during the exercise.
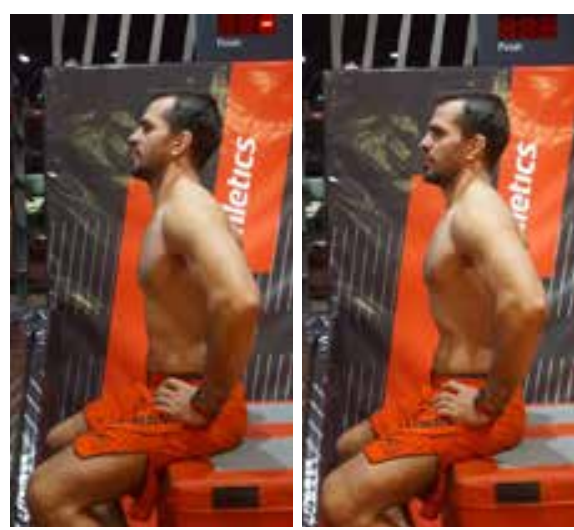

Figure: 5a, 5b: Bracing (5a) with oblique line is nicely visible, breathing is normal versa hollowing $(5 b)$ where the ribcage is suppressed breathing is restricted.

Generally bracing is more effective in stabilizing the lumbar spine as it achieves co-contraction with the LM and other $a b$ dominal muscles like the external oblique in a functional manner. The best way to use the bracing technique is to contract and hold the abdomen like you are waiting for a punch in the stomach and continue to breathe in and out. It is advised to use and practice bracing while sitting, walking, or lying down (Figure 6). The bracing technique can also be used during abdominal and core workouts and it is the ultimate target to achieve with all dynamic exercises. Repeated activation induces plastic changes in the CNS and leads to modified automatic recruitment of the trained muscle during the performance of a functional task (Tsao-Hodges, 2008).

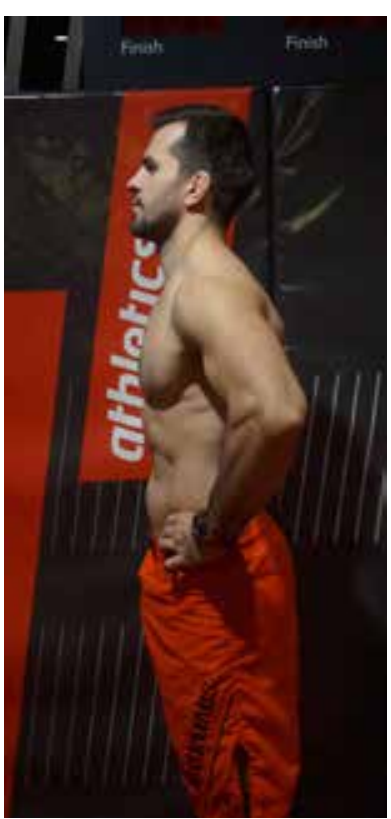

Figure 6: practicing bracing in front of a mirror, client can palpate the $\operatorname{Tr} A$.

Hollowing activates the TrA specifically: contract your abdomen and pull your belly button back toward your spine to make your abdomen as small as possible. Hollowing has its place in training clients and that is at the beginning of training in static positions such as a in lying or in a four point hollowing exercise when we aim to engage only the TrA to its fullest capacity (Hall et al., 2009).

\section{TrA isolation work first}

Initial training can commence in static positions - it may be the only viable option if pain is present. Clients are asked to increase the length of isometric contraction and the repetition numbers, respectively at every session. Subsequently, some external load can be added to more challenging functional body positions, such as a leg or arm lift. More extreme ranges can then be added and finally, co-contractions in dynamic functional movements can then be utilized.

Initially, we can test clients for TrA activation by asking them to pick up an object from the floor. As he or she bends and approaches 45 degrees, the abdominal wall should draw in, and the back and navel should draw closer to one another. If the client's abdomen goes forward or distance remains the same, we can be sure that no TrA activation has happened and that the body is unprotected in forward flexion. From a side view, the stomach begins to bulge forward and the pelvis may rotate forward so that there is an increased lordosis (inward curvature) of the spine. However this can be easily observed in the descending phase of a light squat or in a well under maximum effort deadlift (Figure 7).
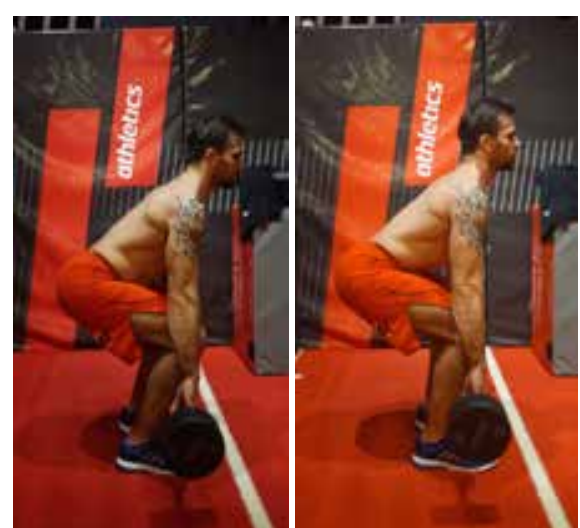

Image: 7a, 7b: Protruding abdomen (7a) versa engaged TrA muscle (7b).

As a rule, for isolation tasks with hollowing, I ask the client to draw in his/her abdominal wall against an air-filled pressure cuff placed under the abdomen. Reduction pressure in the bag can then be measured effectively and evaluate whether the client can activate his or her TrA. (Figure 8). People with history of LBP are unable to perform this maneuver adequately with great enough intensity or long enough, while those with no history have no such issues (Richardson-Jull, 1995). It is in the case of the former, that trainers need to assist clients with physical and verbal pointers. Suggestions I use include having a visual aid to concentrate on when focusing on the pull - showing clients in a mirror how the muscle behaves when contracted, making them palpate the muscle. One can also use helpful phrases that can be easily remembered and repeated for hollowing, such as 'draw the navel towards your spine or draw your lower abdomen up and in', or applying gentle pressure on the area to stimulate it.

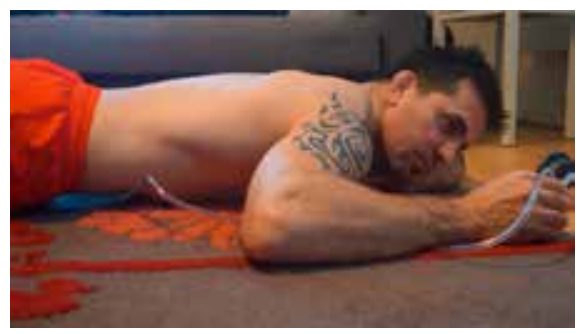

Image 8: Prone abdominal hollowing test and TrA isolation exercise using pressure biofeedback unit. Place the cuff under client's abdomen with the lower edge of the device's cuff just over the line of the pelvis. The unit is then inflated to $70 \mathrm{mmHg}$ and patient is instructed to perform the abdominal hollowing maneuver. The aim is to reduce the pressure reading on the biofeedback unit by 6 to $10 \mathrm{mmHg}$ and to maintain this contraction for 10 repetitions of 10 seconds while breathing normally. (Richardson-Jull, 1995; O'Sullivan et al., 1997) Watch clients ardson-Jull, 1995; O'Sullivan et al., 1997) Watch clients
for common compensations, which include movement of the thoracic and/or lumbar spine or the client presses the knees and feet into the ground.

\section{Progressive exercise selection - Stabiliza- tion training}

Prone position is a great start to training and prompting activation without addition- 
al load. Most people take up this way of activation quite easily and are able to do the exercise even if they have been in pain previously. With the dynamic movements the client activates the abdominal brace before the movement. Its important to emphasis postural alignments with the shoulder blades retracted and depressed. The clients should maintain neutral spine throughout. Progression improves greater resistance/ weight or greater speed.

\section{Abdominal bracing statically}

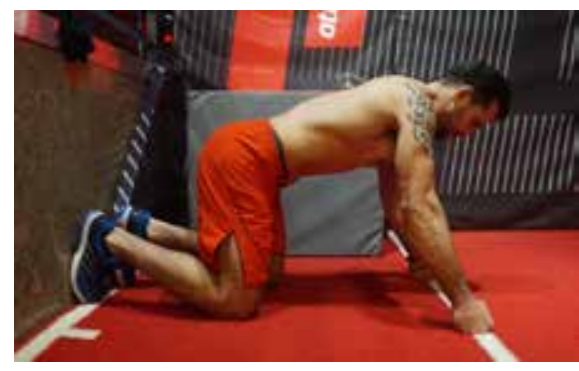

Figure 9. Abdominal bracing: activation of transversus abdominis in four point kneeling.

\section{Abdominal bracing dynamically}

A) In the frontal and horizontal planes

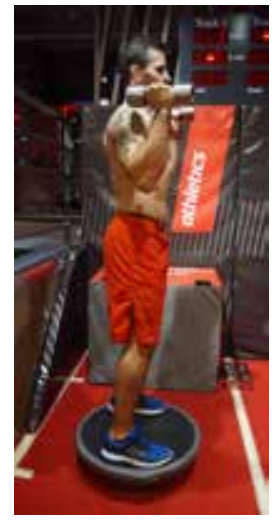

Figure 10. Stand-up position on unstable surface.

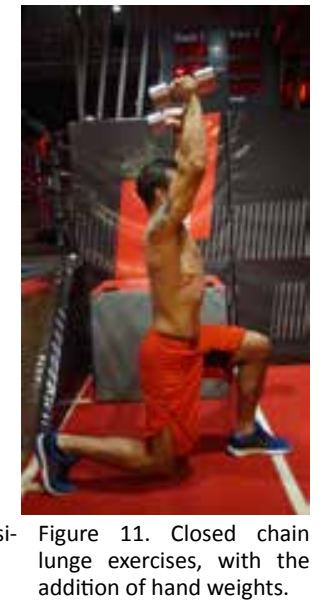

B) In the transvers plane

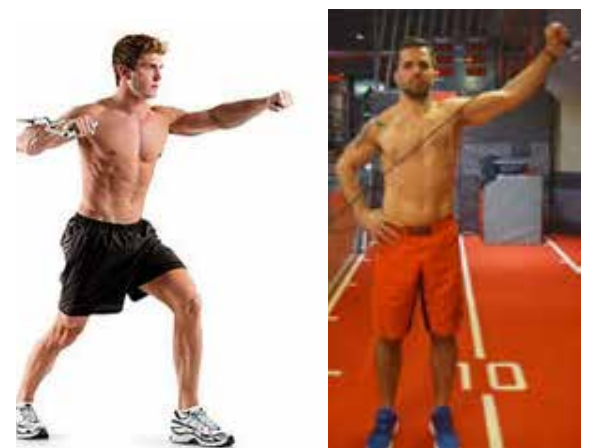

Figure: 12a, 12b: Both exercises requires strict bracing of the abdominals and locking the ribs and pelvis together to avoid unnecessary spinal torsion. The countermovement of the arm in the cable single arm press will decrease rotational forces on the spine.

\section{Summary}

Transversus abdominis is the most important abdominal muscle, it contracts prior to any other abdominal muscle. It provides segmental stability of the spine with the deep back muscle the lumbar multifidus. Furthermore, it should first be trained in isolation, as this will improve its timing of activation and its integration into functional tasks. Foremost, the client needs to learn to activate the muscle lying down in a prone position, next in seated and standing positions, stable and unstable positions and then in the functional dynamic positions also including those where the pain was previously experienced such as walking or getting up.

\section{Conclusions}

Clients need to become aware of the TrA muscle firstly via utilizing palpation, then with help of the pressure cuff method, in static and finally in dynamic movements. Stabilization training programs, which teach the TrA to fire-up prior to movement, is superior to any other strengthening program.

Own experience and research demonstrated, that even just 4 weeks of training but definitely a period of 8-12 weeks of core stabilization training has a preventative effect on low back pain reduction for as long as 6-12 months (Richardson-Jull, 1995; Tsao-Hodges, 2008). But of course the protective effects of training cannot be maintained without one's working environment setup being assessed and an overall good posture being practiced. Conversely, once training has stopped, improvements are not shown and so it is therefore safe to assume that regular (arguably daily) training is required for permanent results.

\section{References}

Akuthota, V. - Ferreiro, A - Moore, T- Fredericson, movement. Experimental Brain Research 124.69-79. DOI: M. (2008): Core stability exercise principles. Current 10.1007/s002210050601

Sports Medicine Reports. 7. 1. 39-44. DOI: 10.1097/01. CSMR.0000308663.13278.69

Cresswell, A.G. - Grundstrom, H. - Thorstensson, A. back pain: a motor control evaluation of transversus ab1992): Observations on intra-abdonimal pressure and dominis. Spine. 21.2640-2650.

patterns of abdominal intra muscular activity in man. Acta Hodges, P.W. - Richardson, C.A. (1997a): Contraction of Physiologica Scandinavica 144. 409-418. DOI: 10.1111/ the abdominal muscles associated with movement of the j.1748-1716.1992.tb09314.x

Cresswell, A.G. - Oddsson, L. - Thorstensson, A. (1994): The influence of sudden perturbations on trunk muscle activity and intra- abdominal pressure while standing. Experimental Brain Research. 98. 336-341. DOI: 10.1007/ BF00228421

Cresswell, A.G. - Thorstensson, A. (1994): Changes in intra-abdominal pressure, trunk muscle activation and force during isokinetic lifting and lowering. Europ. J. Appl. Physiol. 68: 315. doi:10.1007/BF00571450

DeTroyer, A. (1983): Mechanical role of the abdominal muscles in relation to posture. Respiration Physiology. 53. 341-353. DOI:10.1016/0034-5687(83)90124-X

DeTroyer, A. - Estenne, M. - Ninane, V. - VanGansbeke, D. - Gorini, M. (1990): Transversus abdominis muscle function in humans. Journal of Applied Physiology. 68. 1010 1016. DOI: $10.1054 /$ math.1995.0243

Ferreira, P.H. - Ferreira, M.L.- Hodges, P.W. (2004): Changes in recruitment of the abdominal muscles in people with low back pain: ultrasound measurement of muscle activity. Spine. 29. 22. 2560-6. DOI: 10.1097/01 brs.0000144410.89182.f9

França, F.R. - Burke, T.N. - Hanada, E.S - Marques, A.P. (2010): Segmental stabilization and muscular strengthening in chronic low back pain: a comparative study. Clinics. 65 . 10. 1013-1017. DOI: 10.1590/S1807-59322010001000015

Hall, L. - Tsao, H. - MacDonald, D. - Coppieters, M. Hodges, P.W. (2009): Immediate effects of co-contraction training on motor control of the trunk muscles in people with recurrent low back pain. Journal of Electromyography and Kinesiology. 19. 5. 763-73.

Hebert, J.J. - Koppenhaver, S.L. - Magel, J.S. - Fritz, J.M. (2010): The relationship of transversus abdominis and lumbar multifidus activation and prognostic factors for clinical success with a stabilization exercise program: a cross-sectional study. Archives of Physical Medicine and Rehabilitation. 91. 1. 78-85. DOI: 10.1016/j.apmr.2009.08.146.

Hodges, P.W. (1999): Is there a role for transversus abdominis in lumbopelvic stability? Manual Therapy. 4. 2.74 86. DOI: $10.1054 /$ math.1999.0169

Hodges, P.W. - Cresswell, A.G. - Thorstensson, A. (1998): Preparatory trunk motion precedes upper limb

the abdominal muscles associated with move
lower limb. Physical Therapy. 77. 132-144.

Hodges, P.W. - Richardson, C.A. (1997b): Feedforward contraction of transversus abdominis is not influenced by the direction of arm movement. Experimental Brain Research 114. 362-370. DOI: 10.1007/PL00005644

Hodges, P.W. - Richardson, C.A. (1998): Delayed postural contraction of transversus abdominis associated with movement of the lower limb in people with lower back pain. Journal of Spinal Disorders. 11. 46-56. DOI: 10.1097/00002517-199802000-00008

Hodges, P.W. - Richardson, C.A. Archive of Physical Medicine and Rehabilitation. (1999): Altered trunk muscle recruitment in people with low back pain with upper limb movement at different speeds. 80. 9. 1005-12. DOI: 10.1016/S0003-9993(99)90052-7

Kibler, W.B - Press, J.-Sciascia, A. (2006): The role of core stability in athletic function. Sports Medicine. 36. 3 189-98. DOI: 10.2165/00007256-200636030-00001

McArdle, W.D. - Katch, F.I. - Katch, V.L. (1991): Exercise hysiology: Energy, nutrition and human performance. 3rd edition. Lea and Febiger. Phyladelphia. ch20. 384-417.

O'Sullivan, P.B. - Phyty, G.D. - Twomey, L.T. - Allison, G.T. (1997): Evaluation of specific stabilizing exercise in the treatment of chronic low back pain with radiologic diagnosis of spondylolysis or spondylolisthesis. Spine. 22. 24 2959-67. DOI: 10.1097/00007632-199712150-00020

Panjabi M.M. The stabilizing system of the spine. (1992 ): Part I. Function, dysfunction, adaptation, and enhacement. J Spinal Disord. 5: 383-9. DOI: 10.1097/00002517 199212000-00001

Richardson, C.A. - Jull, G.A. (1995): Muscle control-pain control. What exercises would you prescribe? Manual Theraphy. 1. 1. 2-10. DOI: 10.1054/math.1995.0243

Tsao, H. - Hodges, P.W. (2008): Persistence of improvements in postural strategies following motor contro training in people with re-current low back pain. Journal of Electromyography and Kinesiology. 18. 559-67. DOI: 10.1016/j.jelekin.2006.10.012

A.
DOI: 10.1016/j.jelekin.2007.09.008

Title: Personal Trainer, Strength \& Conditioning Coach Munkahelye: Myhealthcare Clinic, Wandsworth Webpage: www.movepainfree.co.uk Levelezési cím: Flat 5, 54b Longthorton Road, Streatham, SW16 50D, London, UK E-mail cime: juditressinkapt@gmail.com Főbb kutatási területei: fájdalommentes mozgás 